\title{
¿Qué considerar al evaluar una dextrocardia por ecocardiografía transtorácica?
}

José Manuel Rivero*

Ceres Moreno**

Luis Martínez ${ }^{* * *}$
Correspondencia

José Manuel Rivero

riverojose24@gmail.com

\footnotetext{
* Servicio de Ecocardiografía. Instituto Diagnóstico Cardiovascular La Plata. Buenos Aires. Argentina

** Servicio de Ecocardiografía. Alberta Children's Hospital. Alberta. Canadá

*** Servicio de Ecocardiografía. Hospital Cardiológico Infantil Latinoamericano Dr. Gilberto Rodríguez Ochoa. Caracas. Venezuela
}

Recibido: 19/08/2020

Aceptado: 25/10/2020

En línea: 31/12/2020

Citar como: Rivero JM, Moreno C, Martínez L. ¿Qué considerar al evaluar una dextrocardia por ecocardiografía transtorácica? Rev Ecocar Pract (RETIC). 2020 (Dic); 3 (3): 43-47. doi: 10.37615/retic.v3n3a3.

Cite this as: Rivero JM, Moreno C, Martínez L. What to consider when evaluating a dextrocardia by transtoracic echocardiography? Rev Ecocar Pract (RETIC). 2020 (Dic); 3 (3): 43-47. doi: 10.37615/retic.v3n3a3.

\section{Palabras clave}

$\triangleright$ Dextrocardia

$\triangleright$ Situs visceral

$\triangleright$ Cardiopatía congénita

Keywords

$\triangleright$ Dextrocardia

$\triangleright$ Visceral situs

$\triangleright$ Congenital heart disease

\section{RESUMEN}

La dextrocardia es una malposición cardíaca que cursa con una orientación del ápex hacia abajo y a la derecha del tórax, acompañada con disposiciones espaciales de las cámaras diferentes según el tipo de situs visceral y la presencia de cardiopatías congénitas asociadas. Por tanto, para la evaluación de estos pacientes se requiere la localización y ejecución de maniobras con el transductor poco convencionales durante la práctica diaria, con el fin de obtener proyecciones ecocardiográficas reproducibles e interpretables que logren una mejor aproximación diagnóstica.

ABSTRACT
Dextrocardia is a cardiac malposition in which the orientation of the cardiac apex is downwards and to the
right side of the thorax, with different spatial dispositions of the chambers according to visceral situs and
associated with other congenital cardiac defects. Therefore for the evaluation of these patients we need to
place and perform non-conventional maneuvers with the transducer during our daily practice, in order to
obtain reproducible and interpretable echocardiographic planes which allow us to reach the best diagnostic
approach.

\section{Introducción}

Las anomalías cardíacas relacionadas con una posición en el mediastino diferente a la levocardia (apéx cardíaco dirigido hacia abajo y a la izquierda) han sido un verdadero reto para su evaluación ecocardiográfica. Por consiguiente, la exploración de una malposición cardíaca como la dextrocardia, definida por la presencia de un corazón preferencialmente en el hemitórax derecho, con una orientación del ápex hacia abajo y a la derecha, puede generar confusión ${ }^{(1)}$. Como consecuencia, han surgido recomendaciones para la adquisición de imágenes, considerando aspectos técnicos y anatómicos, que van a permitir realizar exámenes reproducibles e interpretables. Por esta razón, se explicará de forma simplificada la aproximación ecocardiográfica de esta patología, sin revisar la dextrocardia con anomalías congénitas asociadas o adquiridas concomitantes, ni términos como dextroposición o levoposición, que se relacionan principalmente con afectación extracardíaca (hipoplasia pulmonar, neumotórax, neumonectomía y hernias diafragmáticas), que generan un desplazamiento del corazón hacía el hemitórax derecho o izquierdo, respectivamente, con conservación del eje cardíaco apuntando hacia la izquierda ${ }^{(2)}$.
Esta rara anomalía puede llegar a ser un hallazgo incidental durante una evaluación médica rutinaria, cuando se presenta de forma aislada, o estar asociada a otras cardiopatías congénitas, manifestando signos y síntomas precozmente, siendo esta última detectada principalmente durante la etapa neonatal y primera infancia. No obstante, se han descrito casos que alcanzan la adultez y se presentan con patologías adquiridas como la cardiopatía isquémica y el síndrome aórtico agudo (disección aórtica) (3,4).

Se aconseja que la evaluación de esta cardiopatía congénita se lleve a cabo a través de un enfoque segmentario, donde se identifiquen los segmentos auricular, ventricular y arterial, según sus características morfológicas y no respecto a su ubicación espacial. A su vez, se debe investigar la forma cómo se interrelacionan (conectan) estos segmentos a nivel venoauricular (conexión de venas sistémicas y pulmonares con los atrios), auriculoventricular y ventriculoarterial, además del modo de conexión, que hace referencia a la morfología y estado funcional de las válvulas.

Es importante mencionar que un término clave en la valoración de estos pacientes es el situs visceral, definido como las diferencias (asimetrías) morfológicas, número y distribución de los órganos toracoabdominales en relación 
con la línea media del cuerpo. Se pueden encontrar tres patrones clásicos, situs solitus, situs inversus y heterotaxias (Figura 1), que condicionan a nivel cardíaco:

- La disposición espacial de las cámaras (Figura 2), evidenciando en la dextrocardia con situs solitus o dextroversión, a las cavidades morfológicas derechas ubicadas a la derecha de las cavidades morfológicas izquierdas, contrario a lo que ocurre en un situs inversus o dextrocardia con imagen especular donde las cavidades morfológicas derechas se encuentran a la izquierda de las cavidades morfológicas izquierdas.

- La asociación de cardiopatías congénitas, demostrando mayor prevalencia de múltiples defectos cardíacos cuando la dextrocardia se presenta en situs solitus y ambiguo (isomerismos o heterotaxias) ${ }^{(1,5,6)}$.

\section{¿Qué considerar durante la evaluación?}

Snider et al. ${ }^{(2)}$ sugieren que si el paciente tiene sospecha o signos de dextrocardia, la evaluación debe empezar a partir de la ventana subxifoidea con el paciente en posición supina y el marcador del transductor hacia la izquierda (3 pm), con el haz de ultrasonido orientado hacia el corazón (Figura 3A), ya que esto permite a través de un corte 4 cámaras subxifoideo la confirmación del ápex hacia la derecha (Vídeo 1), con valiosa información sobre la morfología de las cámaras (Figura 4 [situs solitus] y Vídeo 2 [situs inversus]) y la emergencia de las grandes arterias, siempre que la ventana acústica lo permita.

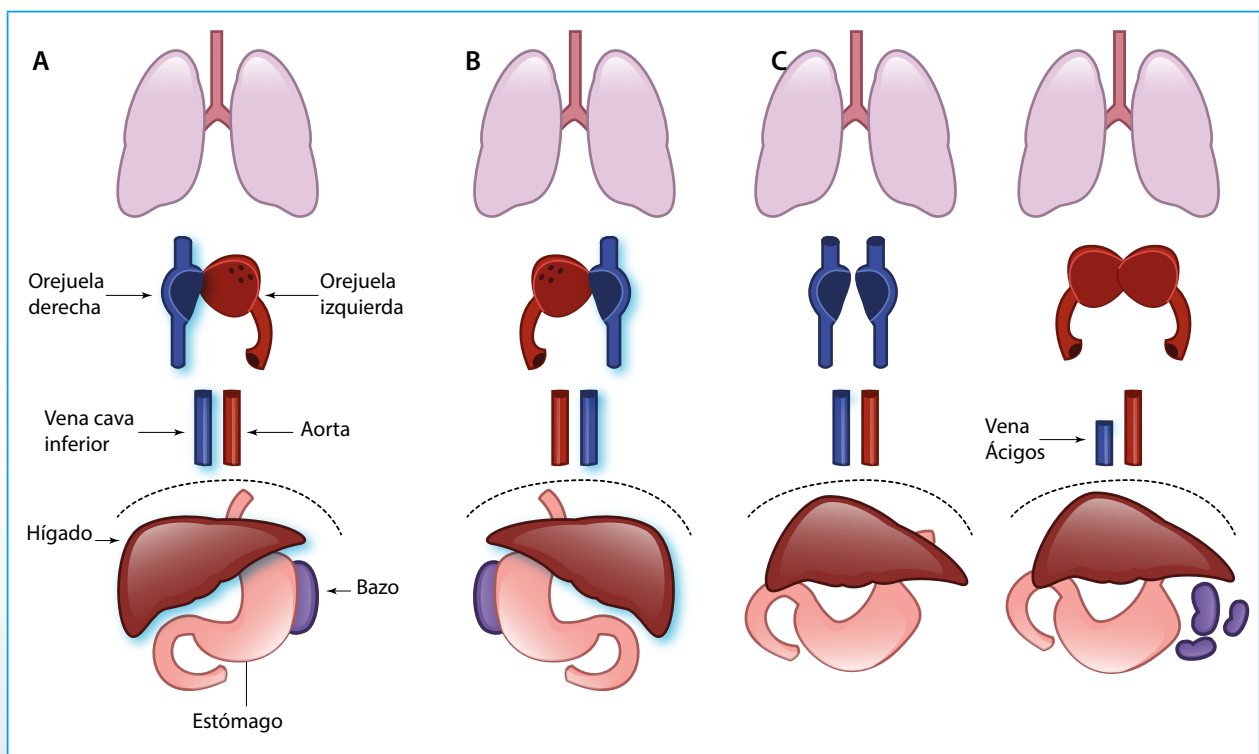

Figura 1. Patrones de situs visceral (diferencias entre la morfología, ubicación y cantidad de los órganos toracoabdominales). A: situs solitus o normal, con la tríada hepato-cavo-atrial a la derecha (órganos con iluminación azul). B: situs inversus totalis o inversión de órganos, con la tríada hepato-cavo-atrial a la izquierda (órganos con iluminación azul). C: situs ambiguo (isomerismos o heterotaxias), relacionado con predominancia de la lateralidad derecha o izquierda del cuerpo (síndromes de asplenia y poliesplenia, respectivamente)

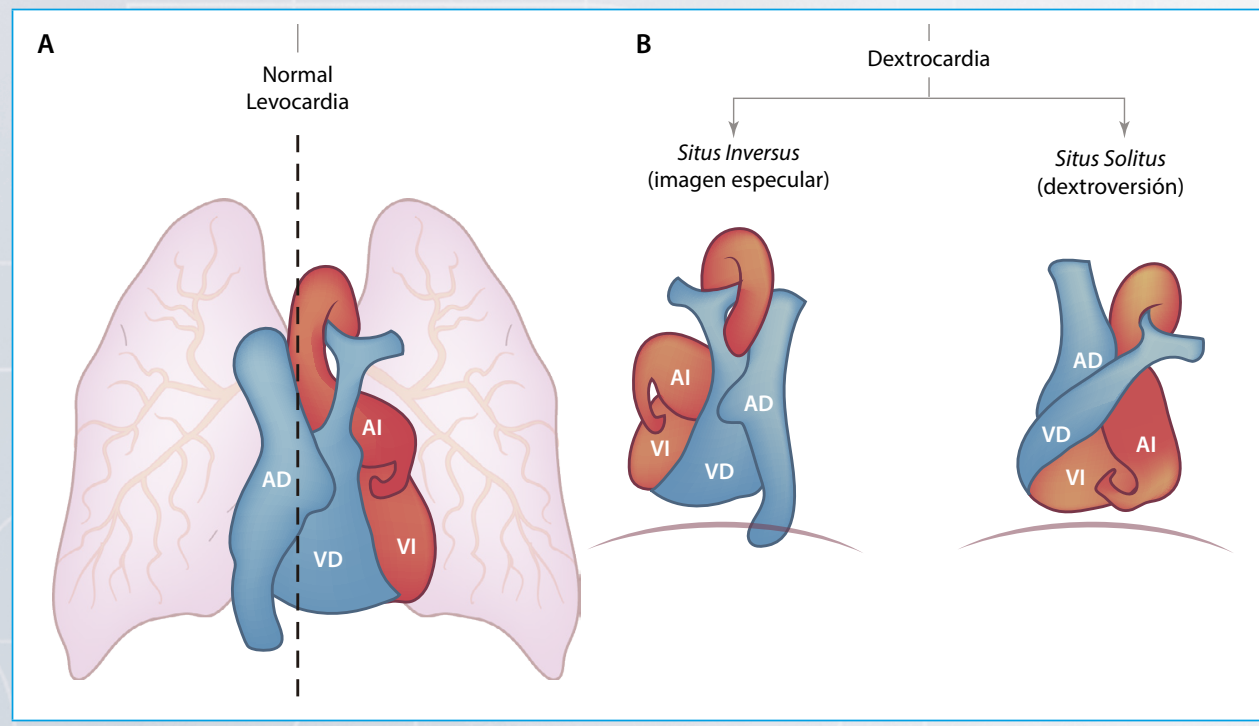

Figura 2.A:posición normal del corazónenel mediastino, con untercio desu volumenala derecha delalínea media (línea negra punteada) y los dos tercios restantes a la izquierda, con el ápex orientado hacia abajo y a la izquierda. B: dextrocardia u orientación del ápex hacia abajo y a la derecha con disposición de las cámaras en función del situs visceral (solitus o inversus) (AD: aurícula izquierda; Al: aurícula izquierda; VD: ventrículo derecho; Vl: ventrículo izquierdo)
A continuación, la determinación del situs visceral es el siguiente aspecto que se ha de precisar. Se ha sugerido que para definirlo se deben localizar estructuras de referencia como el hígado, la porción suprahepática de la vena cava inferior (VCI) y definir el situs auricular (localización de la aurícula morfológica derecha en relación con la línea media del cuerpo), dado que son estructuras muy constantes en su posición e interrelación con los otros órganos, constituyendo la denominada tríada hepato-cavo-atrial. Cuando esta tríada se encuentra a la derecha de la línea media (columna vertebral) con una aorta abdominal (Ao Abd) a la izquierda, se dice que el situs visceral es solitus (Figura 1A), siendo el patrón normal que existe en la mayoría de las personas. No obstante, si la tríada se encuentra a la izquierda con una Ao Abd a la derecha, el situs visceral se denominaría inversus (Figura 1B) ${ }^{(7)}$.

Ahora bien, la visualización de estos hallazgos se logra colocando el transductor desde la misma posición subxifoidea (marcador ubicado a las 3 pm), pero orientando el haz de ultrasonido perpendicular al cuerpo del paciente (Figura 3B); esto permite realizar un corte transverso o axial del abdomen, donde se observa la disposición del hígado a la derecha, VCI anterior y a la derecha de la aorta y Ao Abd. Posterior y a la izquierda en relación con la columna vertebral (Figura 5). Luego, con un giro antihorario del transductor, ubicando el marcador hacia la cabeza del paciente y con el haz orientado ligeramente hacia la derecha (Figura 6), permite visualizar la tríada hepato-cavoatrial con la vena cava ingresando a la AD en el contexto de un situs solitus. Recuérdese que si el situs es inversus, la orientación del haz debe ir hacia la izquierda del paciente. Por su parte, la identificación morfológica de la aurícula derecha se logra evaluando desde múltiples planos ecocardiográficos la forma de su orejuela, la cual tiene un aspecto triangular con base ancha, a diferencia de la orejuela izquierda que es tubular con base estrecha. 


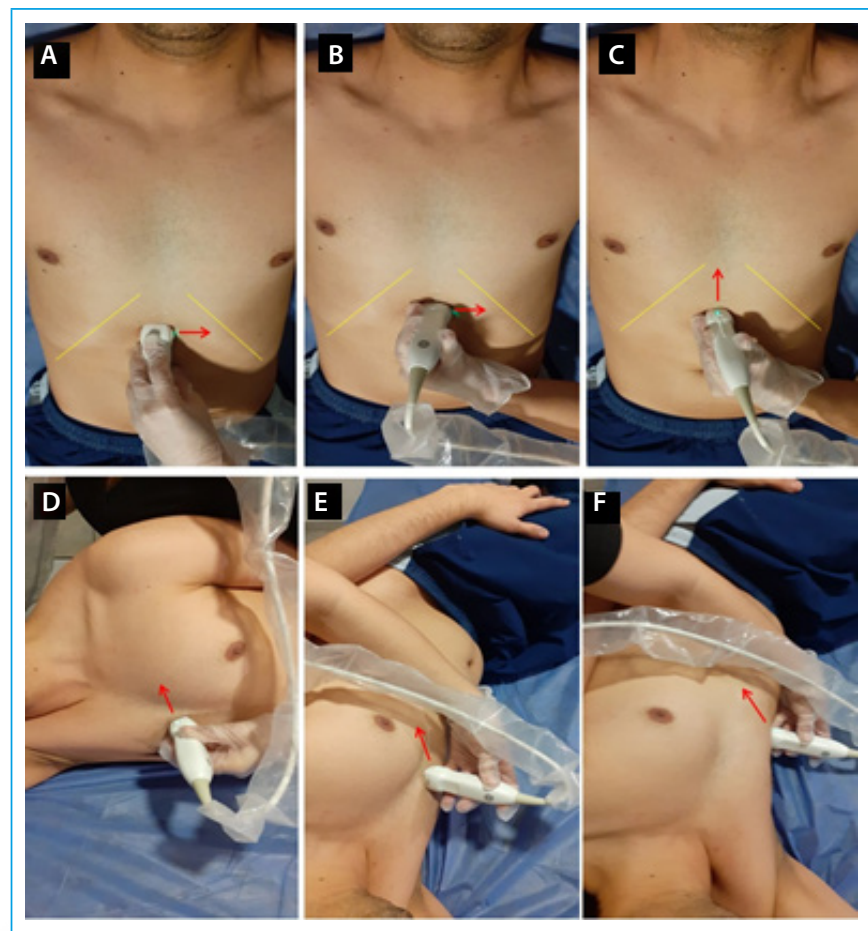

Figura 3. Sitios de colocación del transductor y orientación del marcador (fechas rojas) para llevar a cabo la exploración. Imágenes superiores: ventana subxifoidea (líneas amarillas: arcos costales). A: determinación del ápex hacia la derecha. B: determinación del situs visceral. C: evaluación de la triada hepato-cavo-atrial. Imágenes inferiores: ventana paraesternal y apical derecha. D, E, y F: obtención del eje largo paraesternal, ejes cortos paraesternales y proyecciones apicales, respectivamente

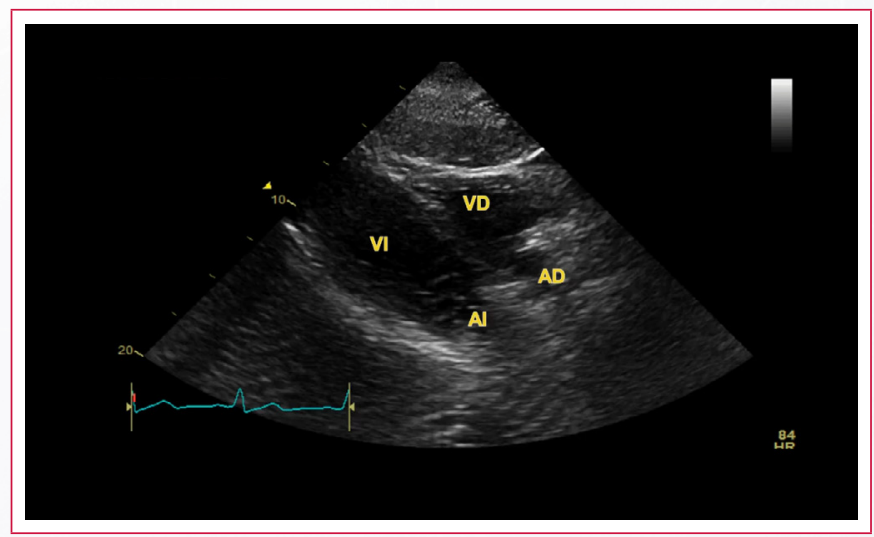

Vídeo 1. Corte subxifoideo de 4 cámaras: ápex a la derecha

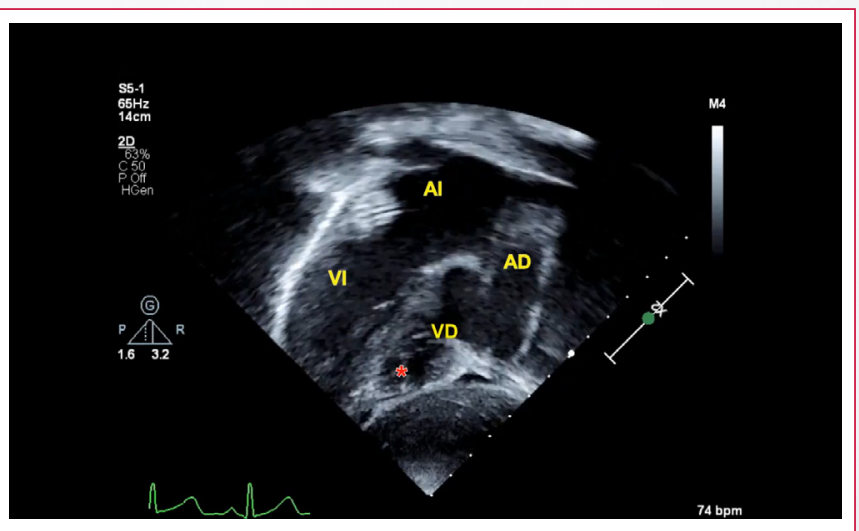

Vídeo 2. Corte subxifoideo de 4 cámaras: situs inversus con dextrocardia
Seguidamente, se procede a obtener imágenes desde la ventana paraesternal. Conviene señalar que muchos de los protocolos de exploración ecocardiográfica comienzan desde esta posición, pero ante la ausencia de visualización de estructuras cardíacas desde la región paraesternal izquierda, se sugiere deslizar o ubicar el transductor entre segundo o tercer espacio intercostal en la región paraesternal derecha, con la orientación del marcador hacia el hombro o región supraclavicular izquierda (Figura 3D), observando un imagen en la pantalla igual a la de un paciente en levocardia, ya que este plano no explora el eje derecha-izquierda del corazón, solamente muestra los ejes anteroposterior e inferosuperior. Se recomienda la colocación del paciente adulto en decubito lateral derecho.

Para hallar el eje corto paraesternal a nivel de las grandes arterias, válvula mitral, músculos papilares y ápex, se debe rotar el transductor desde la misma posición de eje largo paraesternal en sentido horario, con el marcador apuntando a la cadera izquierda del paciente y orientar (angular) el haz ultrasónico hacia arriba o abajo hasta localizar las estructuras de interés (Figura 3E). Un hecho de relevancia es que el situs visceral influye significativamente en la forma como está dispuesto el corazón en sus ejes cortos, ya que en situs inversus (dextrocardia con imagen en espejo) se observa una arteria pulmonar y un ventrículo izquierdo a la izquierda de la pantalla (Figura 7), contrariamente a lo que sucede en situs solitus, donde ventrículo izquierdo y arteria pulmonar se ubicarán a la derecha de la pantalla (como se vería con levocardia).

Por otro lado, para conseguir las vistas apicales, se debe llevar el transductor a nivel del quinto o sexto espacio intercostal, línea axilar anterior derecha o preferiblemente donde se palpe el latido cardíaco, con el marcador orientado hacia la cadera izquierda del paciente (Figura 3F), lo que permite adquirir el plano apical de 4 cámaras y luego, desde allí, se debe rotar el transductor en sentido antihorario para visualizar los planos de 2 y 3 cámaras.

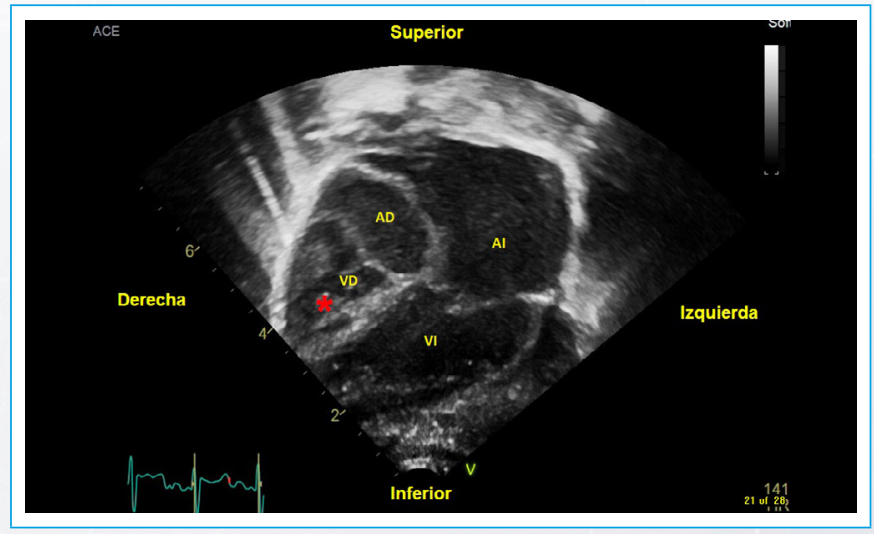

Figura 4. Proyección 4 cámaras subxifoidea en situs solitus (dextroversión), donde las cavidades morfológicas derechas se hallan a la derecha de las cavidades morfológicas izquierdas (lado izquierdo de la pantalla) (asterisco rojo: banda moderadora)

Al igual que en el eje corto paraesternal, el situs visceral vuelve a ser un factor determinante en la forma como se observan las cavidades, especialmente los ventrículos desde la vista apical 4 cámaras (situs ventricular), ya que en situs inversus se localiza un ventrículo morfológico derecho del lado derecho de la pantalla (Vídeo 3) y en el situs solitus se verá a la izquierda de la pantalla (Vídeo 4). Este aspecto es determinado a su vez por los marcadores morfológicos que identifican al ventrículo derecho, dado que posee paredes delgadas con trabéculas prominentes, presencia de la banda moderadora y una valva septal de la válvula tricúspide con una inserción más apical; a diferencia de lo que ocurre en un ventrículo izquierdo donde las paredes son gruesas con menor cantidad trabéculas, no posee banda moderadora y la inserción de la valva anterior de la válvula mitral es más basal. Información relevante para el diagnóstico de cardiopatías congénitas asociadas como la transposición congénitamente corregida de las grandes arterias (ccTGA). 

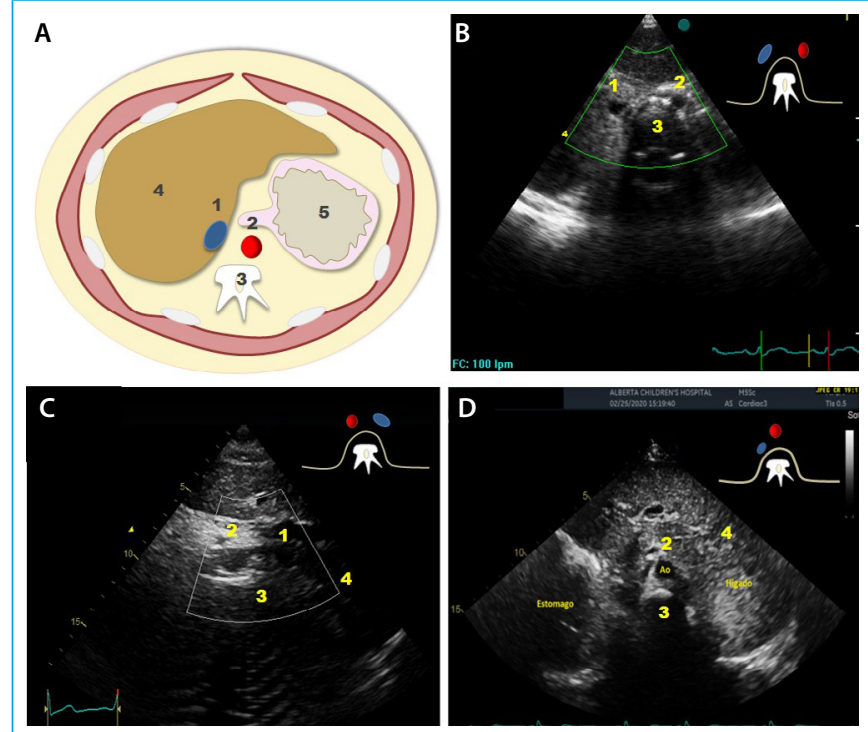

Figura 5. Determinación ecocardiográfica del situs visceral (colocación del transductor: véase Figura 3B). A: representación de un corte transverso del abdomen a nivel subxifoideo, evidenciando la disposición normal de los órganos en situs solitus. Hígado y VCl a la derecha de la columna vertebral, aorta y estómago a la izquierda. B, C y D: patrones ecocardiográficos del situs solitus, inversus y ambiguo, respectivamente. Se determina la ubicación espacial de aorta, $\mathrm{VCl}$ e hígado en relación con la columna vertebral. Obsérvese en la imagen D (isomerismo izquierdo) la posición central de la aorta, asociada a la ausencia del segmento suprahepático de la VCI. La sangre de la porción inferior del cuerpo retorna a la $\mathrm{AD}$ a través del sistema ácigos (1: vena cava inferior [VCI]; 2: aorta; 3: columna vertebral; 4: hígado)

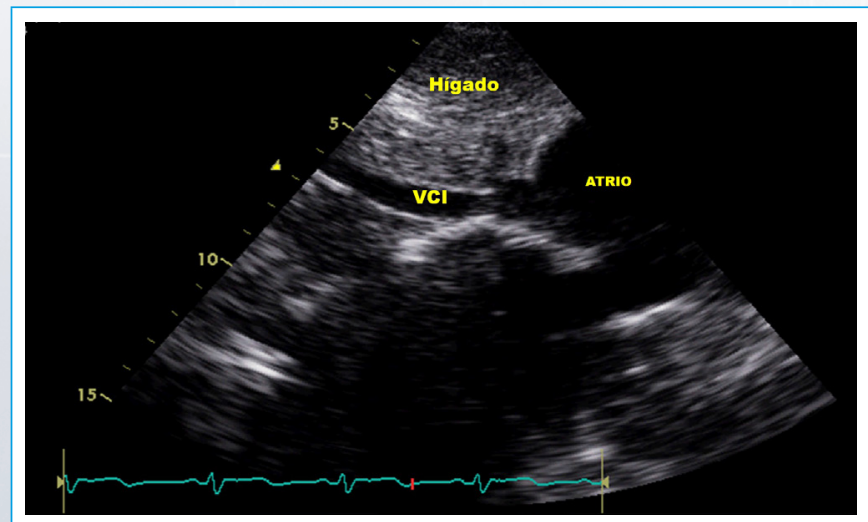

Figura 6. Visualización de la tríada hepato-cavo-atrial (colocación del transductor: véase Figura 3C)

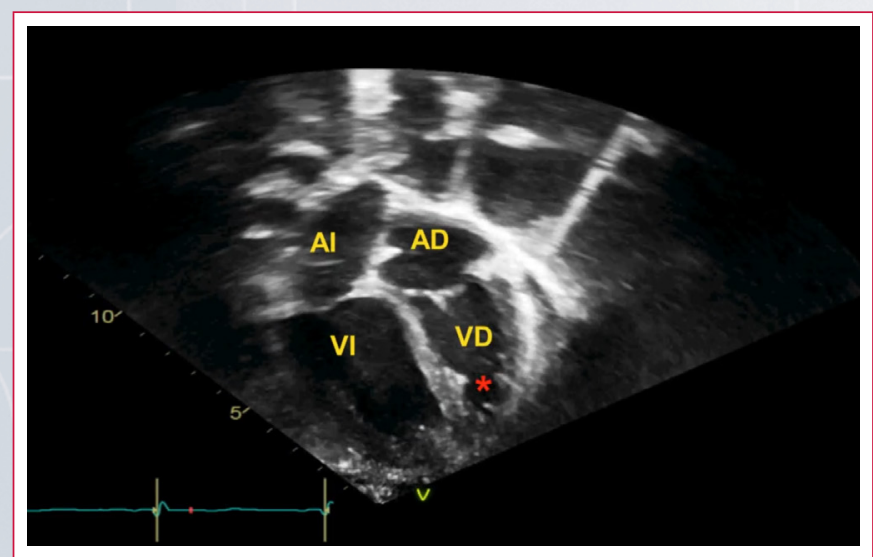

Vídeo 3. Corte apical, 4 cámaras: situs inversus

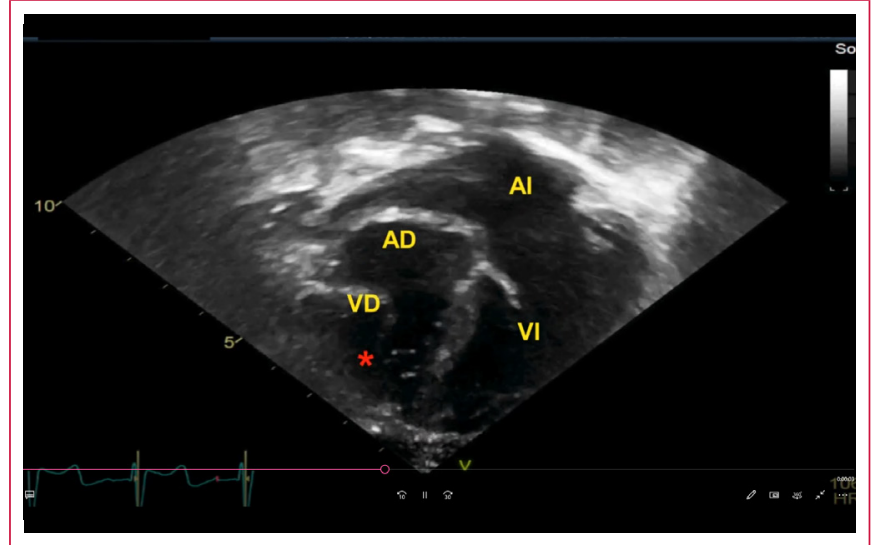

Vídeo 4. Corte apical, 4 cámaras: situs solitus
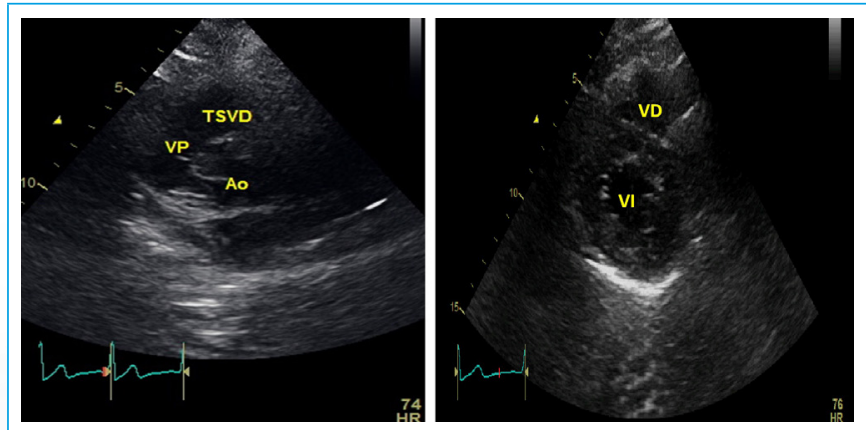

Figura 7. Ejes cortos paraesternales a nivel de las grandes arterias y músculos papilares (colocación del transductor: véase Figura 3E), donde se visualizan la arteria pulmonar y el ventrículo izquierdo a la izquierda de la pantalla, hallazgo sugestivo de dextrocardia con situs inversus (Ao: aorta; TSVD: tracto de salida del ventrículo derecho; VP: válvula pulmonar)

Asimismo, la ventana supraesternal permite evaluar la integridad y orientación del arco aórtico a través de su eje longitudinal, por ende, el transductor debe ir posicionado en la fosa supraesternal con la orientación del marcador dirigida hacia el hombro o fosa supraclavicular izquierda (1 pm) si el arco aórtico se encuentra hacia la izquierda. No obstante, si se sospecha de la presencia de un arco derecho, tal como se esperaría en un situs inversus, el marcador se debe invertir hacia el hombro o fosa supraclavicular derecha (11 am) (Figura 8). Cabe destacar que proyecciones modificadas, como el eje corto de la aorta, también conocida como "vista del cangrejo" (crab view), donde se visualizan las cuatro venas pulmonares conectadas a la aurícula izquierda junto con un corte longitudinal de la vena cava superior, permiten la valoración de anormalidades del drenaje venoso sistémico y pulmonar.

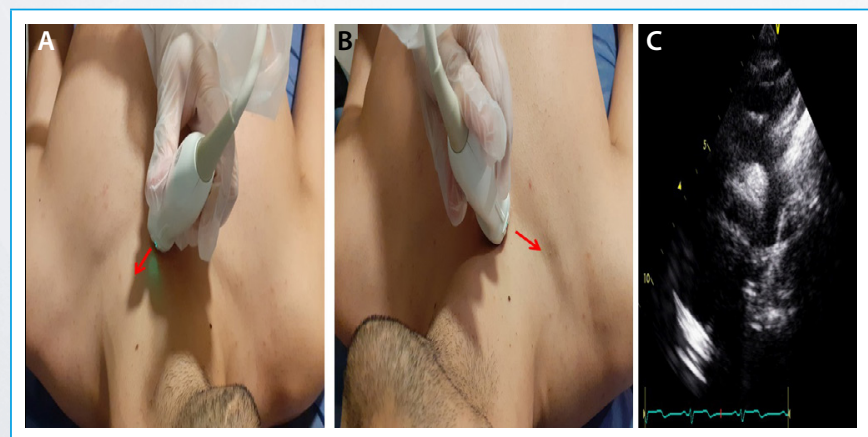

Figura 8. A-B: formas de colocación del transductor desde la ventana supraesternal para la evaluación del arco aórtico izquierdo o derecho, respectivamente (flechas rojas: orientación del marcador del transductor). C: la manera en que se visualiza la disposición del arco en la pantalla no varía independientemente de su localización 
Finalmente, es importante señalar que pueden existir variaciones individuales relacionadas con los sitios específicos de colocación del transductor, así como a la magnitud de maniobras (rotación, deslizamiento e inclinación) que deben realizarse para obtener los planos de imágenes según el contexto clínico del paciente. Un último elemento técnico que se ha de considerar es no cambiar el marcador Izquierda-derecha de la pantalla a través de los controles del equipo, ya que esto tiende a generar confusión al momento de la interpretación, por lo que se recomienda la generación de etiquetas o textos con la finalidad de describir los hallazgos sobre la orientación cardíaca.

\section{Ideas para recordar}

- La disposición de las cavidades cardíacas en pacientes con dextrocardia varía significativamente según el tipo de situs visceral, por lo que su determinación ecocardiográfica se hace primordial durante la evaluación de esta población.

- Se sugiere comenzar el estudio desde la ventana subcostal, ya que confirma del ápex hacia la derecha y conocer el situs visceral.

- La ventana paraesternal y apical se obtiene con el transductor posicionado en el hemitórax derecho en una posición de decúbito lateral derecho.
- Dado que es una malformación que puede coexistir con otras cardiopatías congénitas, se aconseja implementar una aproximación segmentaria, que se enfoque en la morfología de las cámaras, tipos y modos de conexiones.

\section{Bibliografía}

1. Pierre M, Muhamed S. Approach to dextrocardia in adults: review. AJR 2007; 188.

2. Snider R, Serwer G, Ritter S. Echocardiography in pediatric heart diseases, 2nd ed. Mosby, 1997.

3. Abhishek R, Chikkarasinakere M, Kanchanahalli S, et al. Acute myocardial infarction in dextrocardia. A diagnostic and therapeutic challenge. Can dextrocardia be a risk factor? Journal of Cardiology 2018; 17: 48-51.

4. Tetsuya N, Motomi S, Tatsuya I, et al. A case of acute aortic dissection type A in a patient with situs inversus. Ann Thorac Surg 2003; 75: 1963-1965.

5. Robert G. The syndrome of dextroversion of the heart. Circulation 1958; $\mathrm{XVIII.}$

6. Maurice L, Richard L, Friedrich E, et al. Pathologic anatomy of dextrocardia and its clinical implications. Circulation 1968; XXXVII.

7. Anderson R, Baker E, Penny D, Redintowng A, Rigby M, Wenovsky G. Paediatric Cardiology, 3rd ed. Philadelphia. Churchill Livingstone Elsevier, 2010. 